\title{
Smiling, body position, and interpersonal attraction
}

\author{
HUGH McGINLEY, PATSY McGINLEY, and KAREN NICHOLAS \\ University of Wyoming, Laramie, Wyoming 82071
}

\begin{abstract}
Two experiments were conducted. In Experiment 1, regardless of her body position, a woman who smiled the majority of the time $(70 \%)$ was seen as more interpersonally attractive than a woman who seldom smiled $(20 \%)$. When the woman seldom smiled, she was rated as more interpersonally attractive when she displayed open body positions than when she displayed closed body positions. In Experiment 2, the closed body position/smiling and nonsmiling effect was replicated. Subjects' eye gazes were monitored while they viewed the slides of the woman. Regardless of the smiling condition, subjects looked at the woman's face about $55 \%$ of their total looking time.
\end{abstract}

McGinley, LeFevre, and McGinley (1975) and McGinley, Nicholas, and McGinley (1978) found that a female stranger was evaluated more positively when she displayed open as opposed to closed body positions. Although body positions were manipulated, there was no attempt to systematically manipulate other nonverbal information about the stranger.

Given the importance of the face in the expression of emotions, it seems that a valuable extension of the McGinley et al. $(1975,1978)$ studies would be to investigate the concomitant effects of body position and facial expression on interpersonal attraction. Obviously, however, facial expressions per se are multitudinous, and a specific aspect of them, such as smiling, would be a reasonable choice for an initial investigation. ${ }^{1}$

Though smiles can be used for deviated purposes such as deceit (Ekman \& Friesen, 1975), they are more commonly associated with friendliness, happiness, and joy (Izard, 1971). Without discussing the possible innateness of smiling and its inherent relationship to pleasant internal stimuli or to the removal of unpleasant internal stimuli, it seems safe to say that from early infancy humans are exposed to others who smile and that those smiles are usually temporally associated with rewarding interpersonal interactions that satisfy physical and social needs. As a result of these associations, the smile becomes a conditioned stimulus that elicits emotional responses that commonly stimulate evaluative positive overt responses. This interpretation is generally consistent with Lott and Lott's (1972) theory of interpersonal liking.

Empirically speaking, a female stranger who expresses open body positions is seen as more interpersonally attractive than one who displays closed body positions. If smiling elicits positive evaluative responses, the effect of combining smiling and body positions should lead to higher attraction when a person expresses open body positions and smiles a lot than when the person expresses

Requests for reprints should be sent to Hugh McGinley, Department of Psychology, University of Wyoming, Laramie, Wyoming 82071 . closed positions and smiles or when the person smiles little and displays either open or closed body positions.

In the first experiment, subjects were shown slides of a stranger displaying either open or closed body positions and either smiling or not smiling. Experiment 1 prompted the second experiment, in which the subjects' eye-gaze behavior was monitored to see if the stranger's smiling or unsmiling face was attended to differentially:

\section{EXPERIMENT 1}

\section{Method}

Subjects. Ninety-six female students from introductory psychology classes at the University of Wyoming volunteered to participate in the experiment. The subjects met in small groups of from two to seven people.

Materials and Equipment. We used 50 color slides of a model stranger, a Kodak $860 \mathrm{Z}$ projector, two Hunter timers, the Interpersonal Judgment Scale (IJS) (Byrne, 1971), bipolar scales of evaluations, and a postexperimental questionnaire.

The 50 slides consisted of 10 neutral body position slides of the stranger and 10 slides each of open body position/smiling, open body position/nonsmiling, closed body position/smiling, and closed body position/nonsmiling. Two independent judges selected the slides from over 400 taken of the female model stranger. Only slides consistently rated as depicting the stranger in open, neutral, or closed body positions and smiling or not smiling were used. The criterion for rating consistency was that both judges agreed on a slide's rating during an initial and retest judgment. Within the smiling category, body positions were matched (i.e., a body position was presented in both a smiling and nonsmiling mode). The open body slides showed the model in various combinations of the following: leaning backward, legs stretched out, knees apart, one ankle crossed over the other knee, elbows far away from her body, hands held outward, and arms held outward from her body either at her side or elevated. The closed body slides showed that model in some combination of the following: elbows next to her body, arms crossed, hands folded in her lap, knees pressed together, feet together, and legs crossed at either the knees or the ankles. In the 10 neutral slides, the model was typically leaning back, her legs slightly stretched out and slightly apart, hands resting on her lap or on her thighs, and arms held relatively close to her sides.

The IJS (Byrne, 1971) consisted of six seven-alternative items having to do with intelligence, personal liking, working together, personal adjustment, morality, and knowledge of current events. As is typical for attraction research using the IJS, the ratings 
made with the liking and working together items were summed as a single measure of interpersonal attraction. A high score indicated interpersonal attraction (positive evaluation). A secondary measure of attraction was made by having the subjects rate the stranger on four seven-interval bipolar scales of evaluation (sweet-sour, clean-dirty, bad-good, and cruel-kind). The ratings were summed to a single score from 4 (negative) to 28 (positive).

The postexperimental questionnaire consisted of six questions designed to gather information about the subjects' perception of the experiment. The questions were (a) what were you supposed to do; (b) what kind of results do you think we expect to find; (c) were your judgments about the woman based on how you felt about her or on how you thought we expected you to respond (explain); (d) what was the purpose of asking you to make judgments about the woman; (e) specifically, how do you think we expected you to feel about the woman; (f) what other comments do you have?

Procedure. When the subjects arrived at the psychology laboratory, they were met by a female experimenter wearing a white laboratory coat. She introduced herself and explained that the experiment, as the subjects had been told prior to volunteering, involved person perception. The subjects were told that they would receive limited information about a female stranger and that the information was in the form of 20 color slides that had been taken of the woman while she conversed about various topics. The experimenter then told the subjects that they would be asked to make judgments about the woman, based on the information received. The lights were dimmed and the subjects were shown the slides. The slides were shown on a wall screen to a projected size of approximately $120 \times 180 \mathrm{~cm}$. Each slide was shown for $5.5 \mathrm{sec}$; the interslide interval was approximately $.75 \mathrm{sec}$. Following the slide presentation, the subjects made interpersonal judgments about the stranger and filled out the postexperimental questionnaire.

The experimental manipulation was made using four different sets of slides: (a) Open body position/smiling, where 10 neutral body positon slides were interspersed with 10 open body position slides of the stranger. The stranger was smiling in all 10 of the open body posiiton slides and in four of the neutral body position slides. (b) Closed body position/smiling, where the same 10 neutral slides were interspersed with 10 closed body position slides. All of the closed body position slides and four of the neutral body slides showed the stranger smiling. (c) Open body position/nonsmiling, where 10 neutral body position slides were mixed in with 10 open body position slides. None of the open body position slides showed the stranger smiling; however, she was shown smiling in four of the neutral body position slides. The open body positions were matched to those shown in the open body position/smiling condition. (d) Closed body position/nonsmiling, where the 10 neutral slides were interspersed with 10 closed body position slides. None of the closed body position slides showed the stranger smiling. As in the other conditions, the stranger was smiling in four of the neutral body position slides. The body position manipulation, then, consisted of $50 \%$ neutral and $50 \%$ either open or closed body position slides, while the smiling manipulation consisted of either $20 \%$ $(4 / 20)$ or $70 \%(14 / 20)$ smiling across the 20 slides.

Hypothesis. In accord with prior results (McGinley et al., 1975,1978 ), we hypothesized a body position effect where the stranger would be rated as more interpersonally attractive when she expressed open body positions than when she expressed closed body positions. Also, we speculated that the stranger would be evaluated more positively when she smiled frequently than when she seldom smiled. Furthermore, we speculated that with respect to the attraction elicited by the stranger, the order of the experimental conditions from high to low would be smiling-open, smiling-closed, nonsmiling-open, and nonsmilingclosed.

\section{Results and Discussion}

Attraction scores were analyzed by analysis of variance with unequal ns (three subjects' data were omitted because the subjects indicated they knew the stranger). For the IJS score, there was a borderline body position effect $[\mathrm{F}(1,89)=3.61, \mathrm{p}<.07]$ and a smiling effect $[\mathrm{F}(1,89)=33.77, \mathrm{p}<.001]$. However, the Body Position by Smiling interaction was noteworthy $[\mathrm{F}(1,89)=3.66$, $\mathrm{p}<.07$ ] . To avoid a Type II error (which is likely where there is a borderline level of significance for a main effect and a borderline interaction of another variable with that main effect), simple effects analyses were conducted.

Analyses showed that the stranger was rated as most attractive when she smiled; when she smiled a majority of the time, there was no effect for body position. When she smiled very little $(20 \%)$, she was viewed as less attractive. The latter effect, however, was affected by body position; the stranger was seen as less attractive in the closed than in the open body positions. The bipolar scales of evaluation data led to similar findings. There was a significant smiling effect $[F(1,89)=12.42, p<$ $.001]$ and, although there was no main effect for body position, the Body Position by Smiling interaction was significant $[F(1,89)=3.96, p<.05]$. Simple effects analyses for these data led to the same conclusion as did those for the IJS data. Means for both attraction measures are shown in Table 1. In summary, when there was a higher percentage of smiling by the stranger $(70 \%)$, body position did not matter; body position made a difference if she smiled little (20\%). The latter conclusion was supported by the McGinley et al. $(1975,1978)$ finding that a nonsmiling stranger displaying open body positions was seen as more evaluatively positive than on displaying closed positions.

The effect of the smiling variable on the body position variable can be seen in Figures 1 and 2. Two judges read the responses to the postexperimental questionnaire. There was no evidence that the subjects were intuitively aware of the hypothesized relationship between smiling, body position, and interpersonal attraction.

\section{EXPERIMENT 2}

Perhaps, given the relatively short viewing time for each slide $(5.5 \mathrm{sec})$, subjects who saw the smiling stranger were attracted to her smile and spent less time looking at her body and, therefore, were not influenced by body position. The largest difference in the evaluations occurred when the stranger expressed closed positions and

Table 1

Mean Attraction Scores for Body Position and Smiling Conditions, Experiment 1

\begin{tabular}{|c|c|c|c|c|}
\hline \multirow[b]{3}{*}{ Smiling } & \multicolumn{4}{|c|}{ Body Position } \\
\hline & \multicolumn{2}{|c|}{ IJS-Attraction } & \multicolumn{2}{|c|}{ Evaluation-Attraction } \\
\hline & Open & Closed & Open & Closed \\
\hline $70 \%$ & $11.13^{a}$ & $11.13^{\mathrm{a}}$ & $22.75^{a}$ & $22.87^{a}$ \\
\hline $20 \%$ & $9.44 b$ & $7.78^{c}$ & $17.79 b$ & $15.44^{c}$ \\
\hline
\end{tabular}

Note-Values with different superscripts differ at $p<.01$. The standard error of the mean for the IJS-Attraction and Evaluation-Attraction data, respectively, were .44 and .67 . For both sets of data, the higher the score the more positive the attraction. 
either smiled frequently or seldom (Table 1). If these differences were also associated with significant differences in where subjects looked at the stranger, one could conjecture that the body position effect was negated in the smiling conditions, not because of a strong effect of the smiles per se, but because the smiles resulted in the subjects' inattention to the body position. To test this possibility, we monitored the subjects' eye-gaze patterns while they viewed the slides of the stranger.

\section{Method \\ Subjects. Thirty-nine female students from the University of California at San Diego volunteered to serve as subjects. They were obtained through bulletin board advertisement and direct solicitation. The subjects ranged from freshmen to graduate students but were mostly freshmen and sophomores. Each sub- ject was seen individually and was paid $\$ 2$ for her participation. \\ Materials and Equipment. With minor exceptions, the ma- terials and equipment were the same as those used in Experi- ment 1 . The bipolar scales were not used and only the slides from the closed body position/smiling and closed body position/ nonsmiling conditions were used. The slides were projected to a size of approximately $80 \times 120 \mathrm{~cm}$. The slide time was $5.5 \mathrm{sec}$, while the interslide time was $.75 \mathrm{sec}$. Also, a Whittaker Eye View monitor and pupillometer system was used to record the subjects' eye movements. ${ }^{2}$ The eye movement data were re- corded on a Sony 3650 videocorder and then analyzed at a later date. These data were scored for time spent looking at the face of the stranger. The times were recorded in seconds and tenths of seconds and were twice measured by a single judge. The data that were used in the analyses were the average of the two face-viewing times. The interrater coefficient of reliability for the two time measures was .99.}

Procedure. The procedure was the same as in Experiment 1, except that in this experiment the subject was told that her pupillary response would be monitored while she viewed the pictures of the stranger and thought about what kind of person the stranger was. Also, for each subject, the eye monitoring equipment had to be aligned; the alignment took between 2 and $5 \mathrm{~min}$.

\section{Results and Discussion}

The attraction data showed that subjects who saw the closed body position/smiling slides were more interpersonally attracted to the stranger than were those who saw the closed body position/nonsmiling slides. The means were, respectively, 10.61 and $7.95[\mathrm{t}(37)=4.94, \mathrm{p}<$ $.01]$. This result is consistent with that in Experiment 1 .

The looking data were based on 38 subjects, as the data for one subject could not be used. Analysis of these data revealed no difference in face-viewing behavior between subjects who viewed the smiling pictures and those who viewed the stranger smiling very little. On the average, subjects spent about $55 \%$ of their viewing time looking at the stranger's face. Face-viewing times ranged from $30 \%$ to $95 \%$ of the total looking time. Usually, the subjects looked at the stranger's face, scanned across various parts of her body, then looked back at her face.

Although Experiment 2 was conducted as a descriptive study, we thought that a smiling face would attract more visual attention. However, the smiling and nonsmiling faces attracted similar visual attention. Perhaps the subjects modified their eye-gaze behavior because they were aware of our interest in where they looked while viewing the slides. This possibility, however, was not
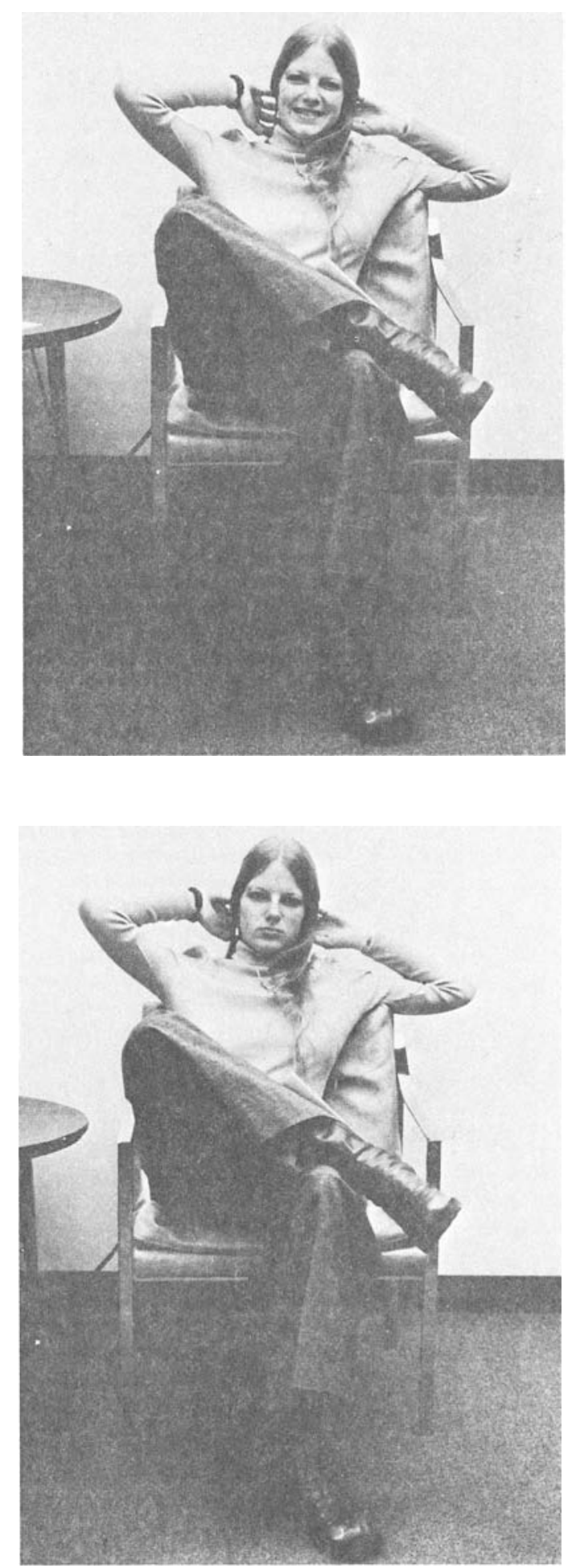

Figure 1. Examples of open body positions with a smiling (a) and nonsmiling stranger (b).

supported by responses to the postexperimental questionnaire. When the experimenter introduced the experimental task to the subjects, he spoke of monitoring the pupil for size changes but did not mention recording eye gaze. A majority of the subjects, in their responses to the postexperimental questionnaire, referred to the rela- 

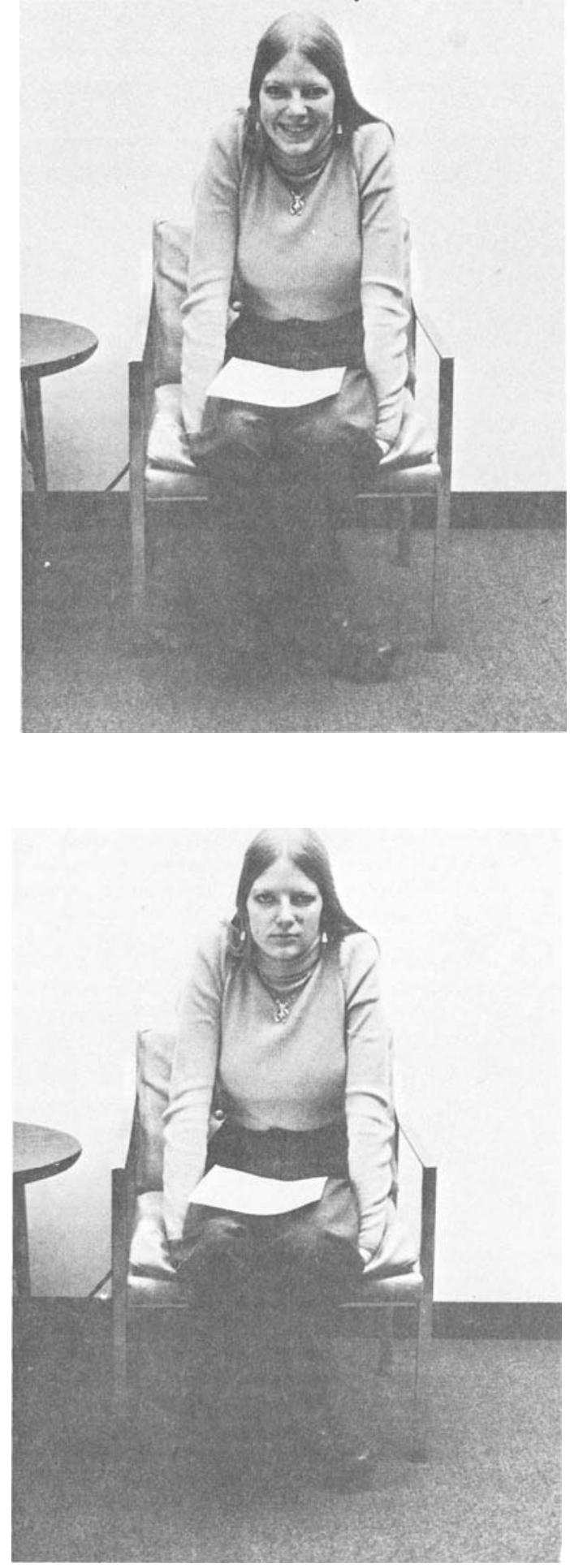

Figure 2. Examples of closed body positions with a smiling (a) and nonsmiling stranger (b). tionship between pupil size and emotional state, but not one mentioned that the experimenter might be interested in where she was looking while viewing the slides.

\section{CONCLUSION}

Although both body position and smiling are aspects of human behavior that frequently and consistently occur during interpersonal interactions, very little is known about their effects on the perceived attraction of the interacting individuals. While the results of the present experiments shed some light on the effects of these variables, the generalizations that can be made from these effects are limited because: (a) the lack of a difference between open and closed body position when the stranger smiled frequently may have been due to a limiting effect of the attraction scale; (b) a single stranger was used and since physical attractiveness is known to be related to interpersonal attraction, the results may have been confounded with the subjects' perceptions of the stranger's physical attractiveness; and (c) only female subjects and a female stranger were used. With these limitations in mind, the results of the two experiments indicate that both smiling and body position influence interpersonal attraction, but that these variables interact such that high smiling negates the attraction effect of body position on interpersonal attraction. Overall, these results warrant the further investigation of the concomitant effects of personal nonverbal behaviors on interpersonal attraction.

\section{REFERENCES}

BYRne, D. The attraction paradigm. New York: Academic Press, 1971.

Ekman, P., \& Friesen, W. V. Unmasking the face. Englewood Cliffs, N.J: Prentice-Hall, 1975.

IzARD, C. E. The face of emotion. New York: AppletonCentury-Crofts, 1971.

Lotr, A. J., \& LotT, B. E. The power of liking: Consequences of interpersonal attitudes derived from a liberalized view of secondary reinforcement. In L. Berkowitz (Ed.), Advances in experimental social psychology (Vol. 6). New York: Academic Press, 1972. Pp. 109-148.

McGinley, H., LeFevre, R., \& McGinley, P. The influence of a communicator's body position on opinion change in others. Journal of Personality and Social Psychology, $1975,31,486-490$.

McGinley, H., Nicholas, K., \& McGinley, P. Effects of body position and attitude similarity on interpersonal attraction and opinion change. Psychological Reports, $1978,42,127-138$.

\section{NOTES}

1. In their 1975 study, McGinley et al. had nearly equal numbers of smiles in their open and closed body position conditions, seven and six respectively. The faces were inked out in the 1978 study.

2. The Whittaker equipment was borrowed from the Navy Personnel Development and Research Center, San Diego, California. Our thanks to Ed Aiken and Tom Duffy for the loan of the equipment. Also, we wish to thank Jean Mandler and the psychology department at the University of California at San Diego for allowing us the use of research facilities.

(Received for publication March 6, 1978.) 